\title{
Interfacial Charge Transfer and Colloidal Semiconductor Dye-Sensitization: Mechanism Assessment via Stark Emission Spectroscopy
}

\author{
Keith A. Walters, Dennis A. Gaal, and Joseph T. Hupp* \\ Department of Chemistry, Northwestern University, 2145 Sheridan Road, Evanston, Illinois 60208-3113 \\ Received: September 13, 2001; In Final Form: April 5, 2002
}

\begin{abstract}
Stark emission spectroscopy has been used to assess the nature of light-induced charge-injection processes occurring at molecular-dye/colloidal-semiconductor interfaces. Experiments reveal that eosin Y injects via a locally excited (LE) state. Coumarin-343 conceivably may react via an interfacial charge transfer state, although a simpler explanation centering on an environmentally perturbed LE state also is consistent with the experimental evidence.
\end{abstract}

\section{Introduction}

Molecule-based sensitization of otherwise transparent, wide bandgap semiconductors to absorption of visible light is a key element of many photoelectrochemical solar energy conversion (light to electricity) schemes. ${ }^{1,2}$ Typically, sensitization is accomplished by exciting the molecular dye to a state sufficiently above the conduction band edge to permit rapid electron transfer to the semiconductor ("injection"). Less commonly, direct photoinjection occurs based on formation of a chromophoric donor(molecule)/acceptor(semiconductor surface) charge-transfer complex. Examples include catechol, ${ }^{3}$ thiocyanate ${ }^{4}$ and ferrocyanide ${ }^{5-7}$ on titanium dioxide surfaces. The two possibilities are illustrated in Scheme 1.

\section{SCHEME 1}
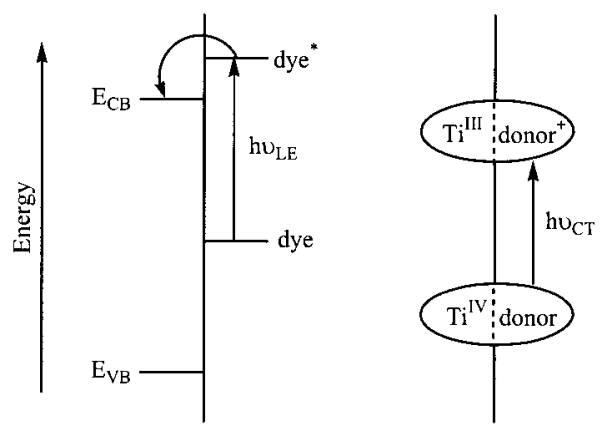

Recently, an intriguing third possibility has been suggested..$^{8-10}$ Binding of a dye to a semiconductor surface may be accompanied by enough electronic interaction such that the locally excited (LE) state of the dye acquires substantial or even dominant interfacial charge transfer (CT) state character. Many examples of LE to CT state interconversion exist in the organic photochemistry literature, ${ }^{11}$ but to our knowledge the idea is new in the context of interfacial photo reactions. The scheme has some potentially advantageous features: (1) the injection yield in the direct CT case is necessarily unity, and (2) by deriving the CT transition from a strongly allowed LE transition, one can expect to obtain much higher absorption cross sections than typically found for pure CT transitions. In addition, as Ghosh has noted, ${ }^{8,9}$ if the CT state is luminescent, the kinetics of back electron transfer can conveniently be measured by time-

*Corresponding author. E-mail: jthupp@chem.nwu.edu. resolving the luminescence (since CT excited to ground state relaxation in this context is identical to back electron transfer).

While the notion of a third sensitization mechanism is clearly appealing, the evidence in support of its occurrence in real dye/ semiconductor systems is largely circumstantial and arguably open to alternative interpretation. We reasoned that a particularly direct test of the mechanism would be to measure the electric field (i.e., Stark) response of the putative CT emission. ${ }^{12,13} \mathrm{~A}$ dominant second-derivative Stark response is the signature of a charge-transfer transition. Furthermore, a quantitative treatment of the Stark signal would be capable of yielding a numerical estimate of the interfacial CT distance. Here we report on Stark emission studies of two of six candidate CT systems suggested in the literature: ${ }^{8,9}$ eosin Y (1) and coumarin-343 (2) on $\mathrm{TiO}_{2}$<smiles>O=C([O-])c1cc(I)ccc1-c1c2cc(Br)c(=O)c(Br)c-2oc2c(Br)c([O-])c(Br)cc12</smiles><smiles>O=C(O)c1cc2cc3c4c(c2oc1=O)CCCN4CCC3</smiles>

colloidal particles. In addition, we describe studies with both dyes on a surrogate surface $\left(\mathrm{ZrO}_{2}\right)$ that is likely to be energetically incapable of functioning as an electron acceptor in the proposed scheme. From the studies reported herein, we find unequivocal evidence that one of the candidate dyes behaves in an LE rather than interfacial CT fashion. The second dye conceivably could possess CT character, although injection via an environmentally perturbed, but otherwise conventional, LE state cannot be ruled out.

\section{Experimental Section}

Sample Preparation. Titanium dioxide ${ }^{14}$ and zirconium dioxide ${ }^{15}$ colloids (10 $\mathrm{nm}$ diameter) were prepared according to the literature. Eosin Y (sodium salt), coumarin-343 and poly(vinyl alcohol) (PVA, $M_{\mathrm{w}}=50 \mathrm{kD}, 99+\%$ hydrolyzed) were obtained from Aldrich and used as received. Ultrapure deionized water was prepared with a Millipore Milli-Q system.

Samples were prepared in PVA films for the Stark emission study to provide an optically clear but polar medium at cryogenic temperatures. Colloid samples $(3 \mathrm{~g} / \mathrm{L})$ were dissolved in ultrapure deionized water, giving a clear solution to which dyes 
were added $\left(1 \times 10^{-5} \mathrm{M}\right.$ for $1,5 \times 10^{-5} \mathrm{M}$ for 2$)$. Solutions were sonicated for one minute, then stirred and gently heated to ensure complete dye dissolution. The solution $\mathrm{pH}$ was maintained at 2.8 to ensure optimal dye adsorption onto the colloidal particles. ${ }^{16}$ PVA was then added $(10 \% \mathrm{w} / \mathrm{w})$ in small increments during vigorous stirring. The solutions were heated and stirred until the PVA completely dissolved and the solution was nearly boiling. Films were cast by pouring the mixture into shallow aluminum dishes and allowing the water to evaporate in a drying cabinet at ca. $50{ }^{\circ} \mathrm{C}$. Film thicknesses were determined by measuring the spacing of IR interference peaks ${ }^{17,18}$ and in all cases were about $190 \mu \mathrm{m}$.

Stark Emission Measurements. The protocol for Stark emission measurements will be described in detail elsewhere. ${ }^{18}$ Briefly, a $2 \times 2 \mathrm{~cm}$ square of sample film was pressed between two ITO-coated glass slides with spring clips and heated at 50 ${ }^{\circ} \mathrm{C}$ for $20 \mathrm{~min}$ to ensure a good electrical contact. The sample "sandwiches" were placed in a liquid $\mathrm{N}_{2}$ immersion dewar with optical windows, and an electric field ( $\mathbf{E}_{\text {external, }}$, typically $2 \times$ $10^{7} \mathrm{~V} \mathrm{~m}^{-1}$ ) was applied. To prevent semiconductor bandgap excitation, $\mathbf{1}$ and $\mathbf{2}$ were excited at 450 and $400 \mathrm{~nm}$, respectively. Emitted light was measured through a horizontal polarizer in a SPEX Fluorolog spectrophotometer using front-face acquisition geometry. Both the constant and delta emission spectra were simultaneously recorded with an SRS 850 digital lock-in amplifier at twice the $200 \mathrm{~Hz}$ AC field modulation frequency. Data were collected at angles $(\chi)$ between the light propagation vector and electric field of $90^{\circ}$ and $62.5^{\circ} .19$

Following standard procedures, ${ }^{12,18}$ the Stark emission spectra were fit, after smoothing (necessary, given the low $\mathrm{S} / \mathrm{N}$ ), to a linear combination of the zeroth, first, and second derivatives of the unperturbed fluorescence spectrum $F(v)$.

$$
\begin{aligned}
& \frac{2 \sqrt{2} \Delta F(v)}{F_{\max }}=\left\{A_{\chi} F(v)+\frac{B_{\chi}}{15 h c}\right. \frac{v^{3} \mathrm{~d}\left[F(v) / v^{3}\right]}{\mathrm{d} v}+ \\
&\left.\frac{C_{\chi}}{30 h^{2} c^{2}} \frac{v^{3} \mathrm{~d}^{2}\left[F(v) / v^{3}\right]}{\mathrm{d} v^{2}}\right\} \mathbf{E}_{\mathrm{int}}{ }^{2}
\end{aligned}
$$

In eq $1, \Delta F(v)$ is the emission change resulting from the electric field modulation, $F_{\max }$ is the maximum intensity of $F(v)$, and $\mathbf{E}_{\text {int }}$ is the internal electric field experienced by the chromophore $\left(\mathbf{E}_{\text {int }}=f \cdot \mathbf{E}_{\text {external }}\right){ }^{20}$ The coefficients $B_{\chi}$ and $C_{\chi}$ provide information about the changes in polarizability and dipole moment, respectively.

$$
\begin{gathered}
B_{\chi}={ }^{5} / 2 \operatorname{Tr} \Delta \alpha+\left(3 \cos ^{2} \chi-1\right)\left({ }^{3} / 2 \hat{\mathbf{g}} \cdot \Delta \alpha \cdot \hat{\mathbf{g}}-{ }_{1}^{1} / 2 \operatorname{Tr} \Delta \alpha\right) \\
C_{\chi}=|\Delta \mu|^{2}\left[5+\left(3 \cos ^{2} \xi-1\right)\left(3 \cos ^{2} \chi-1\right)\right]
\end{gathered}
$$

In eq $2, \operatorname{Tr} \Delta \alpha$ is the trace of the polarizability change between the two states (diagonal components), $\hat{\mathbf{g}} \cdot \Delta \alpha \cdot \hat{\mathrm{g}}$ is the component of the polarizability change along the transition moment ( $\hat{\mathbf{g}}$ is the unit vector), $\Delta \mu$ is the vector dipole moment change, and $\xi$ is the angle between the transition dipole moment and $\Delta \mu$. At least five measurements at both investigated angles were performed on each of three film samples of each dye/ semiconductor colloid combination, fit to eqs 1 and 2 , and the results averaged.

\section{Results and Discussion}

Emission Measurements. Consistent with literature reports, ${ }^{1,8,9}$ we observed substantial absorption and emission red shifts for 2 upon introduction of colloidal particles, but little change for $\mathbf{1}$ (see Figure 1). The spectra shown have been
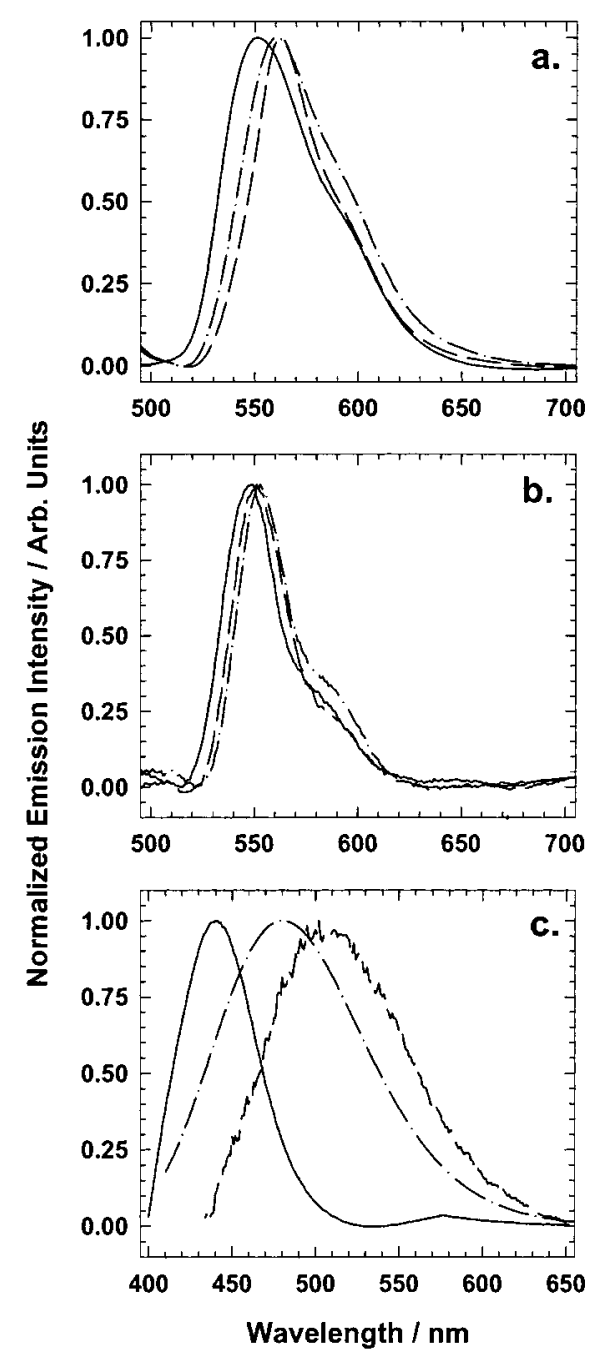

Figure 1. Normalized emission spectra of sample-containing PVA thin films. In all spectra, the solid line is the sensitizer dye by itself, the dashed line is the dye $+\mathrm{ZrO}_{2}$ colloid, and the dotted-dashed line is the dye $+\mathrm{TiO}_{2}$ colloid. (a) $\mathbf{1}, 298 \mathrm{~K}$. (b) $\mathbf{1}, 77 \mathrm{~K}$. (c) $\mathbf{2}, 77 \mathrm{~K}$.

baseline corrected for stray-light contributions. We also observed, consistent with prior reports, that for both dyes $\mathrm{TiO}_{2}$ addition causes the emission quantum yield to decrease-so much so for $\mathbf{2}$ that steady-state emission was only observable at $77 \mathrm{~K}$. One interpretation of this result is that rapid LE-state injection into the semiconductor competes extremely effectively with radiative decay. A second explanation is that the excited state acquires significant interfacial CT character, since CT transitions typically feature larger Franck-Condon factors than LE transitions. ${ }^{11}$ This result often translates into faster nonradiative decay rates and diminished emission quantum yields. A third explanation, at least for $\mathbf{2}$, is that adsorption at the semiconductor nanoparticle surface facilitates intermolecular associations similar to small-molecule $\mathrm{J}$ aggregate formation ${ }^{21}$ a phenomenon that is known to induce spectral red shifts and, in extended oligomeric or polymeric systems, attenuate emission quantum yields. $^{22} \mathrm{~A}$ fourth possibility is that the surface environment alters the $\mathrm{p} K_{\mathrm{a}}$ of the second dye, changing its state of protonation, and thereby changing both its emission spectrum and lifetime.

Introduction of $\mathrm{ZrO}_{2}$ had little effect upon either the energy or emission quantum yield for $\mathbf{1}$ (see Figure 1). Because $\mathrm{ZrO}_{2}$ is characterized by a substantially higher conduction band edge than $\mathrm{TiO}_{2},{ }^{23}$ undiminished (and unshifted) emission would be consistent with formation of an LE state with an energy less 
TABLE 1: Stark Emission Results ${ }^{a}$

\begin{tabular}{|c|c|c|c|c|c|}
\hline \multirow[b]{2}{*}{ sample } & \multicolumn{2}{|c|}{$\lambda_{\mathrm{em}},(\mathrm{nm})^{b}$} & \multirow{2}{*}{$\begin{array}{c}|\Delta \mu| \cdot f^{c} \\
(\mathrm{e} \AA)\end{array}$} & \multirow{2}{*}{$\begin{array}{c}\operatorname{Tr} \Delta \alpha \cdot f^{2, d} \\
\left(\AA^{3}\right)\end{array}$} & \multirow{2}{*}{$\begin{array}{c}\hat{\mathbf{g}} \cdot \Delta \alpha \cdot \hat{\mathbf{g}} \cdot f^{2, e} \\
\left(\AA^{3}\right)\end{array}$} \\
\hline & $298 \mathrm{~K}$ & $80 \mathrm{~K}$ & & & \\
\hline 1 & 556 & 548 & 1.0 & 170 & 260 \\
\hline $\mathbf{1}+\mathrm{ZrO}_{2}$ & 562 & 550 & 0.6 & 60 & 120 \\
\hline $\mathbf{1}+\mathrm{TiO}_{2}$ & 562 & 549 & 0.5 & 60 & 140 \\
\hline 2 & 460 & 437 & 2.3 & 260 & 540 \\
\hline $2+\mathrm{ZrO}_{2}$ & 500 & 500 & 1.7 & 250 & 370 \\
\hline $2+\mathrm{TiO}_{2}$ & $f$ & 470 & 3.9 & 250 & 380 \\
\hline
\end{tabular}

${ }^{a}$ All measurements recorded on PVA films at $77 \mathrm{~K}$. Estimated uncertainties in $|\Delta \mu| \cdot f$ values, based on replicate measurements, are $\pm 10 \% .{ }^{b}$ Emission maximum. ${ }^{c}$ Product of vector change in dipole moment and local field correction parameter. ${ }^{d}$ Product of the trace of the change in polarizability and the square of the local field correction parameter. ${ }^{e}$ Product of the vector change in polarizability and the square of the local field correction parameter. ${ }^{f}$ Emission too weak to record.

than that of the $\mathrm{ZrO}_{2}$ conduction band, making injection unfavorable. We found that binding of 2 to $\mathrm{ZrO}_{2}$, on the other hand, caused a substantial red shift in emission energy, exceeding the red shift caused by $\mathrm{TiO}_{2}$. Similar behavior has been reported by Ghosh. ${ }^{8}$ If predominantly CT-like excited states were present on both colloidal surfaces, the more negative bandedge for $\mathrm{ZrO}_{2}$ should have yielded an emission that was blueshifted with respect to the emission from $2+\mathrm{TiO}_{2}$. The reversed energy ordering (see Table 1) argues, but does not prove, that at least one of the observed emissive states is not CT in nature. ${ }^{24}$ A further difference for $\mathbf{2}+\mathrm{ZrO}_{2}$ versus $\mathbf{2}+\mathrm{TiO}_{2}$ is the occurrence of less emission quenching for the former. The occurrence of only modest quenching likewise argues against a CT assignment for photoexcited $\mathbf{2}+\mathrm{ZrO}_{2}$ but could be compatible either with J-aggregate formation or with a change in state of protonation, as suggested above. ${ }^{22}$ Dye $\mathbf{2}$ is known to aggregate in water, ${ }^{8}$ and both dyes are only sparingly soluble in aqueous solutions (the medium used for dye loading).

Stark Emission Measurements. Representative Stark emission spectra for $\mathbf{1}+\mathrm{TiO}_{2}$ and $\mathbf{2}+\mathrm{TiO}_{2}$ are shown in Figures 2 and 3 , respectively. The top panel of each figure corresponds to the corrected field-independent emission spectrum, while the second panel displays its second derivative. The third and fourth panels of each contain Stark emission spectra collected at $\chi=$ $90^{\circ}$ and $62.5^{\circ}$, respectively. The Stark emission spectra were fit using eqs 1 and 2 . The fits yielded little zeroth derivative component. The average percentages, taking all spectra into account, for the three components were $\sim 0.2$ (zeroth derivative), $\sim 14$ (first derivative), and $\sim 86$ (second derivative). Note that fits conducted with only two parameters (i.e., omitting the $A_{\chi}$ term in eq 1) produced essentially identical fit parameters for $B_{\chi}$ and $C_{\chi}$. These findings, along with the similar shapes of the unperturbed emission second derivative and the recorded Stark signal, indicate that changes in the dipole moment account for the majority of the observed signal. Note that because of the weak emission intensity and resulting low $\mathrm{S} / \mathrm{N}$ for these measurements, Stark spectral fits (for all samples) were confined to the peak region, as indicated in the figures. Table 1 summarizes the Stark parameters obtained for all samples. In the absence of nanoparticles, both dyes display small but finite changes in dipole moment upon excited-state to ground-state conversion concomitant with emission. These changes are not unexpected and simply reflect modest internal charge redistribution associated with LE state relaxation. As shown in the table, dye attachment to $\mathrm{ZrO}_{2}$ results in a modest $|\Delta \mu| \cdot f$ decrease for both dyes ( 0.4 and $0.6 \mathrm{e} \cdot \AA$, respectively). Neither is consistent with emission from an interfacial CT state.

Turning to $\mathrm{TiO}_{2}$, the $|\Delta \mu| \cdot f$ value for dye $\mathbf{1}$ exhibits a decrease similar to that observed for $\mathrm{ZrO}_{2}$ attachment. We conclude that

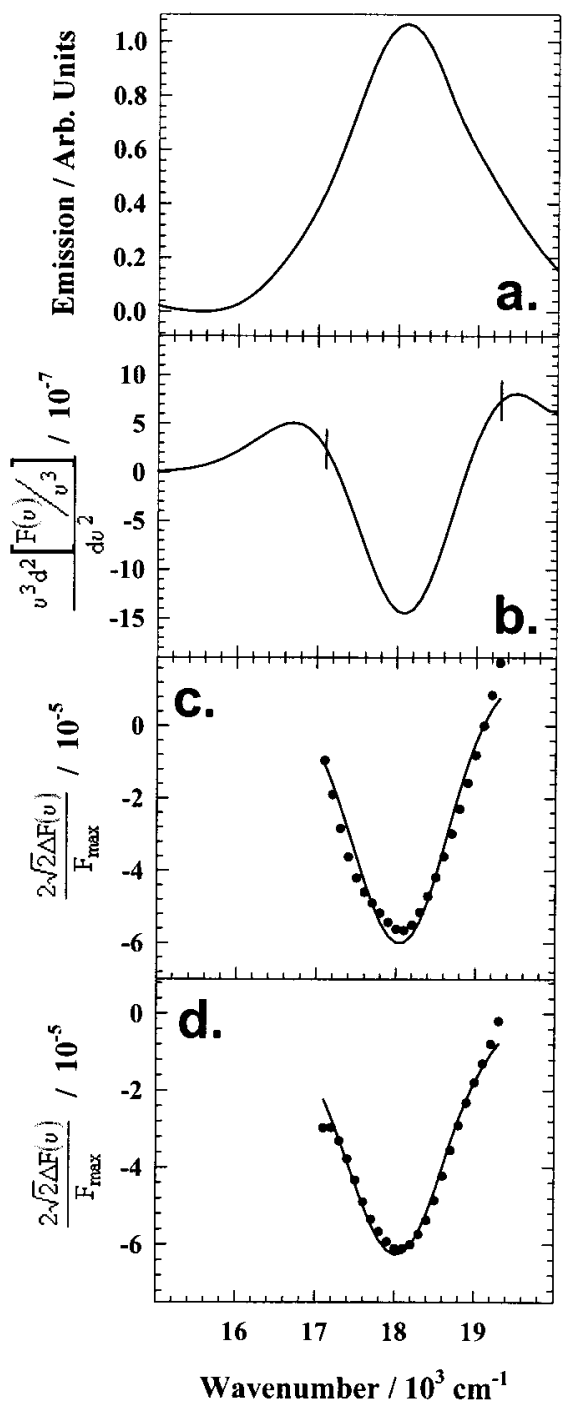

Figure 2. Stark emission spectra of $\mathbf{1}+\mathrm{TiO}_{2}$ colloid in a PVA film. (a) Baseline-corrected emission spectrum (smoothed to permit derivative assessment). (b) Second derivative of emission spectrum. (c) $\chi=90^{\circ}$ Stark emission signal (dots) and experimental fit (line). (d) $\chi=62.5^{\circ}$ Stark emission signal (dots) and experimental fit (line).

1 both injects and emits from a conventional LE state (Scheme 1). For dye $2,|\Delta \mu| \cdot f$ increases by $1.6 \mathrm{e} \cdot \AA$ upon attachment to $\mathrm{TiO}_{2}$. We conclude that for this system emission conceivably could be occurring from an interfacial CT state of the kind envisioned by Ghosh, ${ }^{8,9}$ although a more prosaic explanation centering on aggregation ${ }^{25}$ or surface environmental effects cannot be ruled out. If the speculation concerning interfacial CT state formation is correct, then the overall dipole moment change $( \pm 3.9 \mathrm{e} \cdot \AA)$ between the two colloidal systems can be viewed as the effective distance for semiconductor-to-dye back electron transfer (ET). The distance is consistent with back ET from a site at the edge of the nanoparticle since the colloidal particles are $10 \mathrm{~nm}$ in diameter. The relatively small distance compared to the particle size suggests that back ET occurs via a localized trap state rather than the semiconductor conduction band, which would exhibit a larger ET distance due to the ability of the electron to migrate away in the conduction band from the hole on the dye.

\section{Conclusions}

Mechanisms of semiconductor colloid dye sensitization have been studied using Stark emission spectroscopy. We believe 


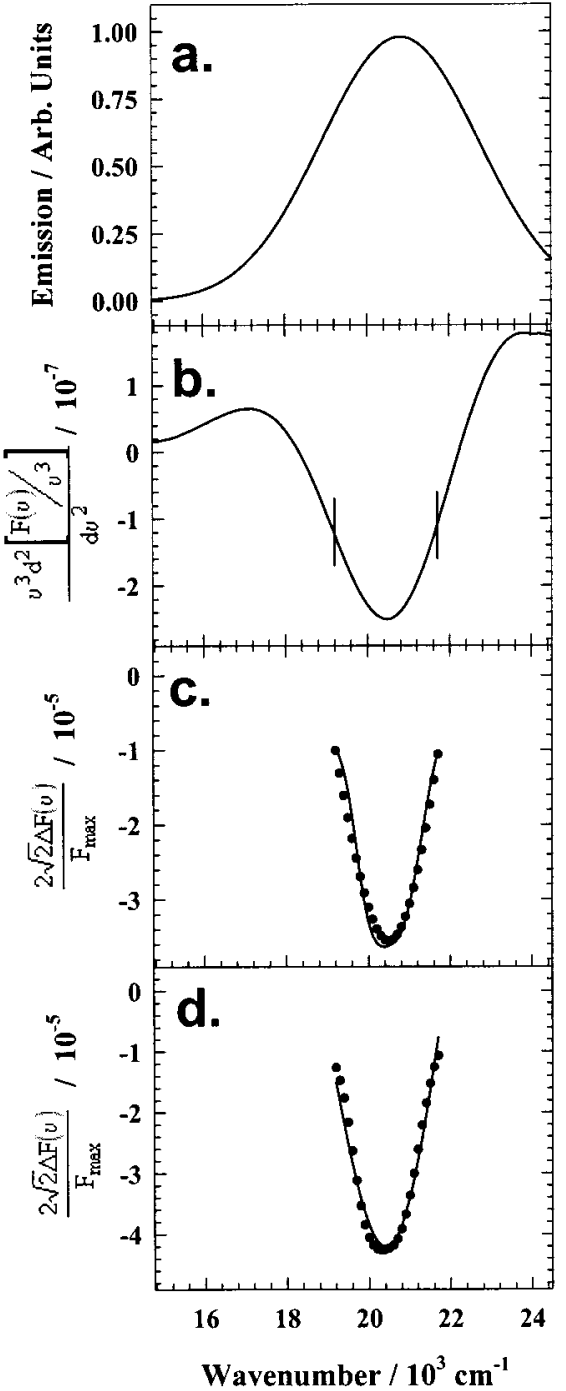

Figure 3. Stark emission spectra of $\mathbf{2}+\mathrm{TiO}_{2}$ colloid in a PVA film. (a) Baseline-corrected emission spectrum (smoothed to permit derivative assessment). (b) Second derivative of emission spectrum. (c) $\chi=90^{\circ}$ Stark emission signal (dots) and experimental fit (line). (d) $\chi=62.5^{\circ}$ Stark emission signal (dots) and experimental fit (line).

this is the first application of this technique to interfacial chargetransfer processes. ${ }^{26}$ The studies reveal that eosin $\mathrm{Y}$ sensitizes $\mathrm{TiO}_{2}$ via injection from an LE state. Coumarin-343, on the other hand, conceivably may inject from a state featuring significant interfacial CT character, although a simpler alternative explanation centering on injection from an environmentally perturbed LE state also is consistent with the experimental evidence.

Acknowledgment. We gratefully acknowledge financial support from the U.S. Department of Energy, Office of Science (Grant DE-FG02-87ER13808).

\section{References and Notes}

(1) Moser, J.; Grätzel, M. J. Am. Chem. Soc. 1984, 106, 6557-6564

(2) (a) Gaal, D. A.; Hupp, J. T. J. Am. Chem. Soc. 2000, 122, $10956-$ 10963. (b) Kamat, P. V.; Meisel, D. Semiconductor Nanoclusters-Physical, Chemical, and Catalytic Aspects; Elsevier: Amsterdam, 1997; Vol. 103. (c) Frank, A. J.; Gregg, B. A.; Grätzel, M.; Nozik, A. J.; Zaban, A.; Ferrere, S.; Schlichthörl, G.; Huang, S. Y. In Future Generation Photovoltaic Technologies; McConnell, R. D., Ed.; American Institute of Physics: Woodbury, 1997; pp 145-153. (d) Huang, S. Y.; Schlichthörl, G.; Nozik, A. J.; Grätzel, M.; Frank, A. J. J. Phys. Chem. B 1997, 101, 2576-2582. (e) Hagfeldt, A.; Grätzel, M. Chem. Rev. 1995, 95, 49-68. (f) Linsebigler, A. L.; Lu, G.; Yates, J. T. Chem. Rev. 1995, 95, 735-758. (g) Bard, A. J.; Fox, M. A. Acc. Chem. Res. 1995, 28, 141-145.
(3) (a) Persson, P.; Bergstroem, R.; Lunell, S. J. Phys. Chem. B 2000 , 104, 10348-10351. (b) Moser, J.; Punchihewa, S.; Infelta, P. P.; Grätzel, M. Langmuir 1991, 7, 3012-3018. (c) Redmond, G.; Fitzmaurice, D.; Grätzel, M. J. Phys. Chem. 1993, 97, 6951-6954.

(4) (a) Rossetti, R.; Beck, S. M.; Brus, L. E. J. Am. Chem. Soc. 1982 104, 7322-7324. (b) Bahnemann, D.; Henglein, A.; Lilie, J.; Spanhel, L. J. Phys. Chem. 1984, 88, 709-711.

(5) (a) Vlachopoulos, N.; Liska, P.; Augustynski, J.; Grätzel, M. J. Am. Chem. Soc. 1988, 110, 1216-1220. (b) Vrachnou, E.; Grätzel, M.; McEvoy, A. J. J. Electroanal. Chem. 1989, 258, 193-205.

(6) (a) Blackbourn, R. L.; Johnson, C. S.; Hupp, J. T. J. Am. Chem. Soc. 1991, 113, 1060-1062. (b) Doorn, S. K.; Blackbourn, R. L.; Johnson, C. S.; Hupp, J. T. Electrochim. Acta 1991, 36, 1775-1785. (c) Lu, H.; Prieskorn, J. N.; Hupp, J. T. J. Am. Chem. Soc. 1993, 115, 4927-4928.

(7) (a) Ghosh, H. N.; Asbury, J. B.; Weng, Y.-X.; Lian, T. J. Phys. Chem. B 1998, 102, 10208-10215. (b) Weng, Y.-X.; Wang, Y.-Q.; Asbury, J. B.; Ghosh, H. N.; Lian, T. J. Phys. Chem. B 2000, 104, 93-104.

(8) Ghosh, H. N. J. Phys. Chem. B 1999, 103, 10382-10387.

(9) Ramakrishna, G.; Ghosh, H. N. J. Phys. Chem. B 2001, 105, 70007008 .

(10) Yet another mechanism is reductive quenching of the dye by the redox mediator, followed by thermal electron transfer from the dye to the semiconductor. For an example, see: Thompson, D. W.; Kelly, C. A Farzad, F.; Meyer, G. J. Langmuir 1999, 15, 650-653.

(11) Jones, G. In Photoinduced Electron Transfer; Fox, M. A., Chanon, M., Eds.; Elsevier: Amsterdam, 1988; Vol. A, pp 245-305. 250.

(13) (a) Lockhart, D. J.; Goldstein, R. F.; Boxer, S. G. J. Chem. Phys. 1988, 89, 1408-1415. (b) Lockhart, D. J.; Hammes, S. L.; Franzen, S.; Boxer, S. G. J. Phys. Chem. 1991, 95, 2217-2226.

(14) Lyon, L. A.; Hupp, J. T. J. Phys. Chem. 1995, 99, 15718-15720.

(15) Xu, Q.; Anderson, M. A. J. Mater. Res. 1991, 6, 1073-1081.

(16) Grätzel, M.; Moser, J. J. Am. Chem. Soc. 1984, 100, 6557-6564.

(17) Karki, L.; Hupp, J. T. Inorg. Chem. 1997, 36, 3318-3321.

(18) Walters, K. A.; Kim, Y. J.; Hupp, J. T. Inorg. Chem., in press.

(19) The second angle takes into account the refraction of the incident radiation through the ITO cell, see: Chowdury, A. C.; Locknar, S. A.; Premvardhan, L. L.; Peteanu, L. A. J. Phys. Chem. A 1999, 103, 96149625.

(20) The local field correction factor, $f$, is reported to be 1.11 for a spherical molecule in similar polymer matrixes. (See: Ponder, M.; Mathies, R. J. Phys. Chem. 1983, 87, 5090-5098.) Because the dye environment here is more complex, comprising both PVA and metal-oxide interactions, and because dipolar effects not included in the continuum estimate for $f$ (PVA) could conceivably be significant, we have chosen not to attempt the correction. Parameters instead are reported as $\Delta \mu \cdot f, \operatorname{Tr} \Delta \alpha \cdot f^{2}$, and $\hat{\mathbf{g}}$. $\Delta \alpha \cdot \hat{\mathrm{g}} \cdot f^{2}$

(21) Maiti, N. C.; Mazumdar, S.; Periasamy, N. J. Phys. Chem. B 1998, $102,1528-1538$

(22) (a) Samuel, I. D. W.; Rumbles, G.; Collison, C. J.; Moratti, S. C.; Holmes, A. B. Chem. Phys. 1998, 227, 75-82. (b) Rumbles, G.; Samuel, I. D. W.; Collison, C. J.; Moratti, S. C.; Holmes, A. B. Synth. Met. 1999, 101, 158-161. (c) Rumbles, G.; Samuel, I. D. W.; Magnani, L.; Murray, K. A.; Demello, A. J.; Crystall, B.; Moratti, S. S.; Stone, B. M.; Holmes, A. B.; Friend, R. H. Synth. Met. 1996, 76, 47-51. (d) Rughooputh, S. D. D. V.; Hotta, S.; Heeger, A. J.; Wudl, F. J. Polym. Sci. B. Polym. Phys. 1987, 25, 1071-1078.

(23) (a) Kay, A.; Humphry-Baker, R.; Grätzel, M. J. Phys. Chem. 1994, 98, 952-959. (b) Tachibana, Y.; Moser, J. E.; Grätzel, M.; Klug, D. R.; Durrant, J. R. J. Phys. Chem. 1996, 100, 20056-20062. (c) Cherepy, N. J.; Smestad, G. P.; Grätzel, M.; Zhang, J. Z. J. Phys. Chem. B 1997, 101, 9342-9351.

(24) Studies by Lindqvist and Shakhverdov on eosin-line xanthene dyes, for example, show significant emission spectral shifts as the sample medium pH is altered. See: Martin, M. M.; Lindqvist, L. J. Lumin. 1975, 10, 381390 and Maslov, V. G.; Turaeva, Z. N.; Shakhverdov, T. A. Zh. Anal. Khim. 1986, 41, 622-628.

(25) Previous Stark studies of small organic dyes have shown that J-type aggregate formation can induce small changes in $|\Delta \mu|$. Typically, the values increase (see, for example: Gottfried, D. S.; Boxer, S. G. J. Lumin. 1992 51, 39-50. Beekman, L. M. P.; Steffen, M.; van Stokkum, I.; Olsen, J. D.; Hunter, C. N.; Boxer, S. G.; van Grondelle, R. J. Phys. Chem. B 1997, 101, 7284-7292. Stanley, R. J.; Jang, H. J. Phys. Chem. A 1999, 103 , 8976-8984). These studies also report, however, significant increases in $\Delta \alpha$, which are not observed here.

(26) We are aware, however, of preliminary work by B. S. Brunschwig, A. R. Parise, and N. Sutin on the application of Stark absorption spectroscopy to interfacial electron-transfer reactions (Proceedings of the 24th DOE Solar Photochemistry Research Conference, June, 2001, p 87). 